\title{
Treatment of Chronic Pulmonary Aspergillosis: Current Standards and Future Perspectives
}

\author{
Ana Alastruey-Izquierdo ${ }^{a}$ Jacques Cadranel ${ }^{b, c}$ Holger Flick ${ }^{d}$ \\ Cendrine Godet ${ }^{\mathrm{C}}$ Christophe Hennequin ${ }^{\mathrm{f}, \mathrm{g}}$ Martin Hoenigl ${ }^{\mathrm{h}, \mathrm{i}}$ \\ Chris Kosmidis ${ }^{j}$ Christoph Lange ${ }^{k-n}$ Oxana Munteanu ${ }^{\circ}$ lain Page ${ }^{j}$ \\ Helmut J.F. Salzer ${ }^{k, l}$ on behalf of CPAnet
}

\begin{abstract}
a Mycology Reference Laboratory, National Centre for Microbiology, Instituto de Salud Carlos III, Madrid, Spain; bService de Pneumologie, AP-HP, Hôpital Tenon, Paris, France; ' Sorbonne Université, Paris, France; d Division of Pulmonology, Department of Internal Medicine, Medical University of Graz, Graz, Austria; 'Service des Maladies

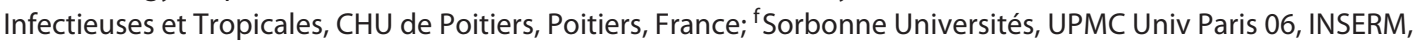
Centre de Recherche Saint-Antoine (CRSA), Paris, France; 9 Service de Parasitologie-Mycologie, AP-HP, Hôpital Saint-Antoine, Paris, France; ${ }^{\text {h}}$ Division of Infectious Diseases, University of California, San Diego, CA, USA; 'i Section of Infectious Diseases and Tropical Medicine and Division of Pulmonology, Medical University of Graz, Graz, Austria; jNational Aspergillosis Center, Manchester University Foundation Trust, UK, and Division of Infection, Immunity and Respiratory Medicine, Faculty of Biology, Medicine and Health, Manchester Academic Health Science Centre, The University of Manchester, Manchester, UK; ${ }^{2}$ Division of Clinical Infectious Diseases, Research Center Borstel, Leibniz Lung Center, Borstel, Germany; 'German Center for Infection Research, Clinical Tuberculosis Center, Borstel, Germany; ${ }^{\mathrm{m} I n t e r n a t i o n a l ~ H e a l t h / I n f e c t i o u s ~ D i s e a s e s, ~ U n i v e r s i t y ~ o f ~ L u ̈ b e c k, ~ L u ̈ b e c k, ~ G e r m a n y ; ~}{ }^{\mathrm{n}}$ Department of Medicine, Karolinska Institutet, Stockholm, Sweden; ${ }^{\circ}$ Division of Pneumology and Allergology, Department of Internal Medicine, State University of Medicine and Pharmacy Nicolae Testemitanu, Chisinau, Moldova
\end{abstract}

\section{Keywords}

Chronic pulmonary aspergillosis · Aspergillus · Antifungal treatment · Management

\begin{abstract}
Chronic pulmonary aspergillosis (CPA) complicates conditions including tuberculosis, chronic obstructive pulmonary disease and sarcoidosis, and is associated with high morbidity and mortality. Surgical cure should be considered where feasible; however, many patients are unsuitable for surgery due to extensive disease or poor respiratory function. Azoles are the only oral drug with anti-Aspergillus activity and itra-
\end{abstract}

\section{KARGER}

(c) 2018 S. Karger AG, Basel

E-Mail karger@karger.com

www.karger.com/res conazole and voriconazole are considered as first-line drugs. A randomized controlled trial demonstrated improvement or stability in three-quarters of patients given 6 months of itraconazole, but a quarter relapsed on stopping therapy. Long-term treatment may therefore be required in some cases. Itraconazole, voriconazole and posaconazole require therapeutic drug monitoring. No published data are yet available for isavuconazole. Adverse drug effects of azoles are common, including peripheral neuropathy, heart failure, elevated liver enzymes, QTc prolongation and sun sensitivity. Many serious drug-drug interactions occur, including major interactions with rifamycins, simvastatin, warfarin, clopidogrel, immunosuppressant drugs like sirolimus. Further- 
more, drug resistance occurs, including cross-resistance to all azoles, but the true prevalence is not yet determined. Intravenous therapy is possible with echinocandins or amphotericin $B$, but long-term use is challenging. Hemoptysis complicates CPA and can be fatal. Tranexamic acid should be given acutely to reduce bleeding. Bronchial artery embolization can stop acute bleeds. In some circumstances, emergency surgery may be necessary to resect the source of the bleed. Current CPA treatments can be beneficial but have many drawbacks. New oral anti-Aspergillus agents are needed, along with optimization of currently available treatments.

c 2018 S. Karger AG, Basel

\section{Introduction}

Chronic pulmonary aspergillosis (CPA) is characterized by slowly progressive destruction of lung parenchy$\mathrm{ma}$, in the form of single of multiple cavities, nodules, infiltrates or fibrosis, with or without an aspergilloma (Fig. 1, 2). It affects patients with preexisting pulmonary pathology such as active or previous tuberculosis (Fig. 3) and nontuberculosis mycobacterial infection, chronic obstructive pulmonary disease, sarcoidosis, or previous surgery for lung cancer [1]. Due to the nonspecific and indolent presentation, diagnosis may be delayed or missed, resulting in severe morbidity. Diagnostic criteria include presence of respiratory and/or constitutional symptoms for at least 3 months, suggestive abnormalities on imaging, and serological or microbiological evidence of Aspergillus [2].

Antifungal therapy can lead to improvement of symptoms and radiological findings (Fig. 4), although a considerable proportion of patients do not respond to treatment [3]. To date, no commonly accepted treatment outcome definition has been defined. It has to be considered that no common treatment endpoints have been defined for CPA. A randomized controlled trial demonstrated improvement or stability in three-quarters of patients given 6 months of itraconazole, but a quarter relapsed on stopping therapy. Long-term treatment may therefore be required in some cases. Voriconazole produced similar results in a single-arm prospective trial, and posaconazole use has been described in a case series. Relapses occur often after treatment is stopped, in approximately one-third of cases [4]. Consequently, patients usually remain on long-term, often lifelong, antifungal therapy, and there are no widely accepted criteria or endpoints for treatment discontinuation $[3,5]$. In addition, toxicity of antifungal therapy is significant, and development of resistance during therapy is common in more extensive disease, e.g. bilateral disease with aspergillomas [6]. Surgery offers a chance of cure, but this is limited to patients with a single aspergilloma or with nonprogressive CPA, localized dis-
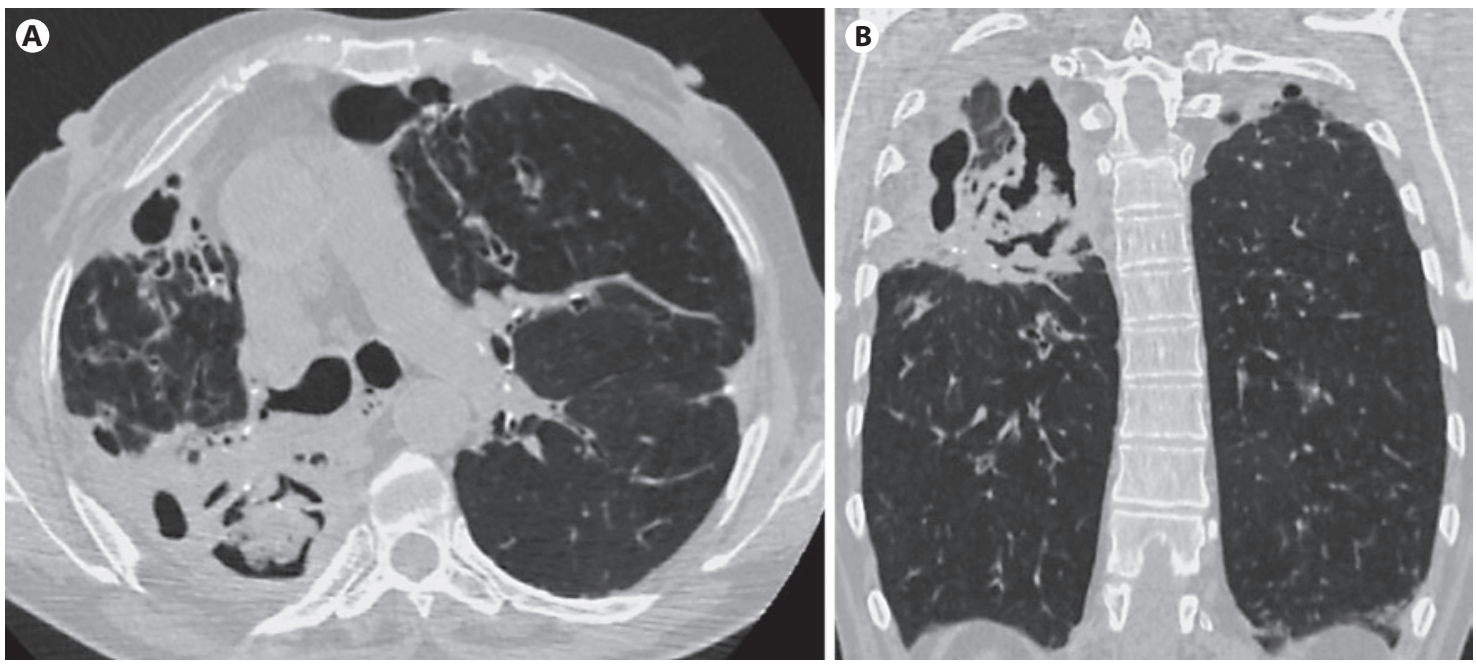

Fig. 1. CT scan of the chest of a 55-year-old woman with chronic cavitary pulmonary aspergillosis presenting with severe hemoptysis and a past medical history of pulmonary tuberculosis 16 years ago. Axial (A) and coronal (B) CT scans of the chest show a large irregular cavity containing a fungus ball with pleural thickening of the right upper lobe. Smaller cavities are shown in the apical section of the left lobe. 
ease, and no significant comorbidities. Finally, the number of antifungal drug classes is small, and the triazoles are the only class of oral drugs available. Research into new classes of antifungals that can be administered by the oral route in patients with CPA is urgently needed. This review will briefly discuss the current approach to CPA management.

\section{Which Antifungal Treatment Should Primarily Be Used?}

Triazoles including itraconazole and voriconazole are the treatment of choice with AII recommendation in the guideline published by the European Society for Clinical Microbiology and Infectious Diseases (ESCMID), the Eu-
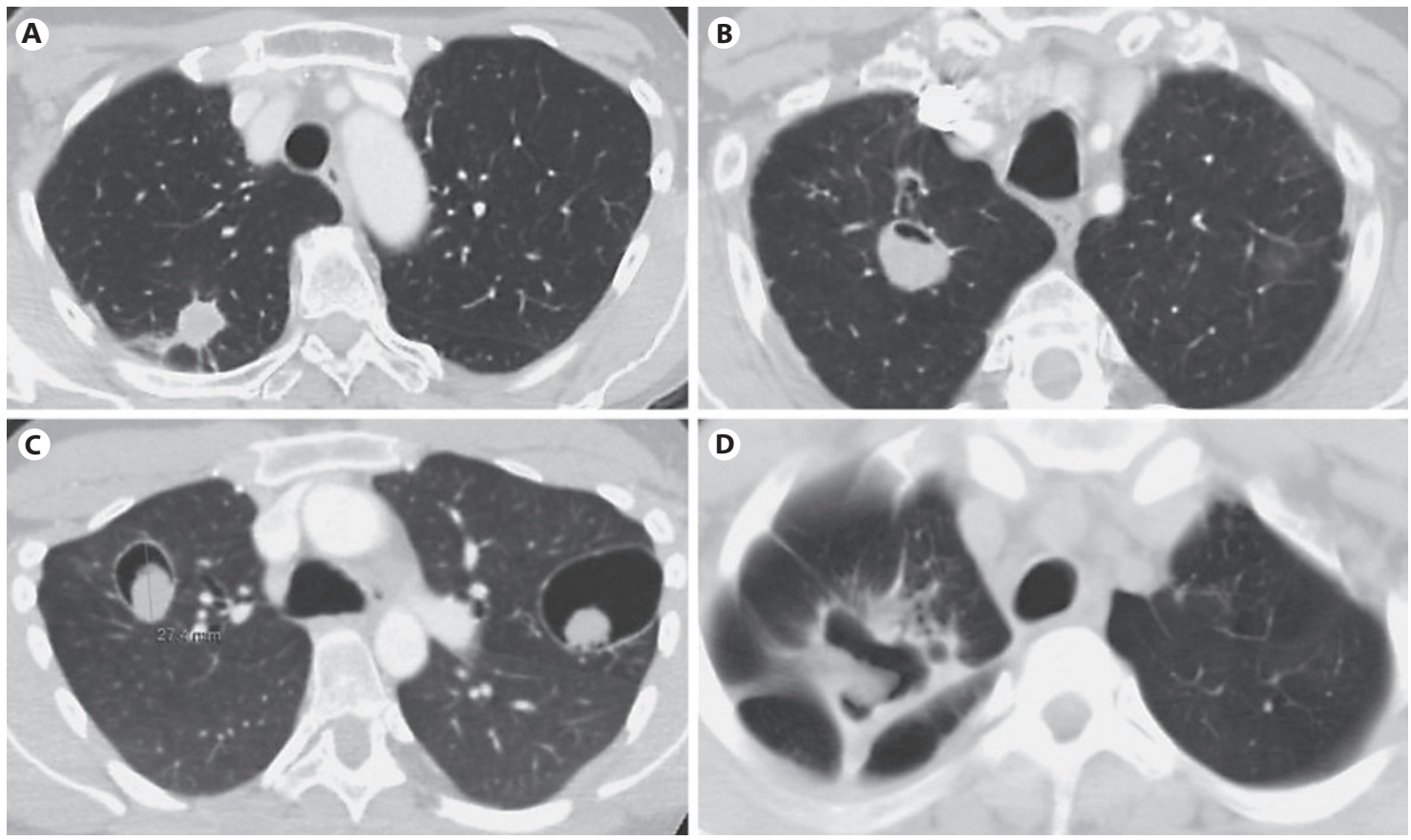

Fig. 2. CT scans of the chest from patients with chronic pulmonary aspergillosis. Aspergillus nodules (A), unilateral simple aspergilloma that is amenable to surgery (B), bilateral cavities with aspergilloma (C), making surgical resection challenging, thick-walled chronic cavitary pulmonary aspergillosis (D).

Fig. 3. A Coronal CT scan of the chest showing multiple bipulmonary large fungal balls in a 69-year-old man with residual cavities from prior pulmonary tuberculosis. Severe bronchiectasis is found in both lower lung fields. B A 37-year-old man with prior pulmonary tuberculosis with a large residual postoperative pleural space and bronchopleural fistula. Two years ago, he underwent lobectomy of the left upper lobe due to life-threatening hemoptysis. Coronal CT scan of the chest shows a marked pleural thickening with irregular cavitary wall containing a mass.
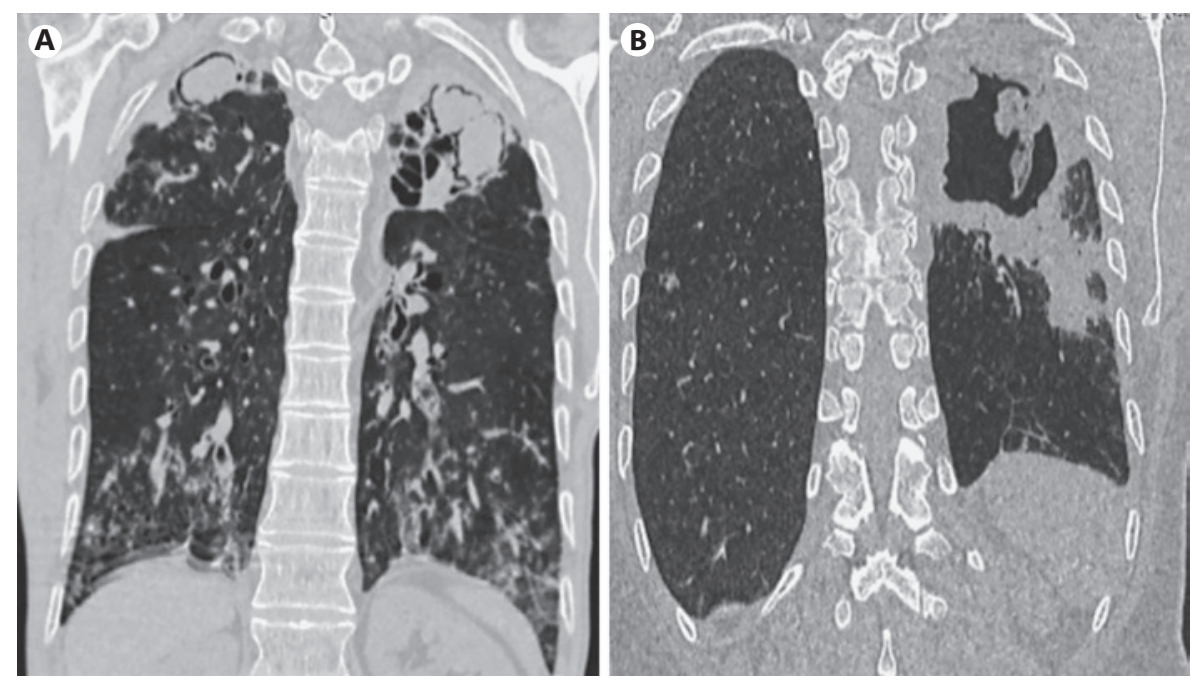


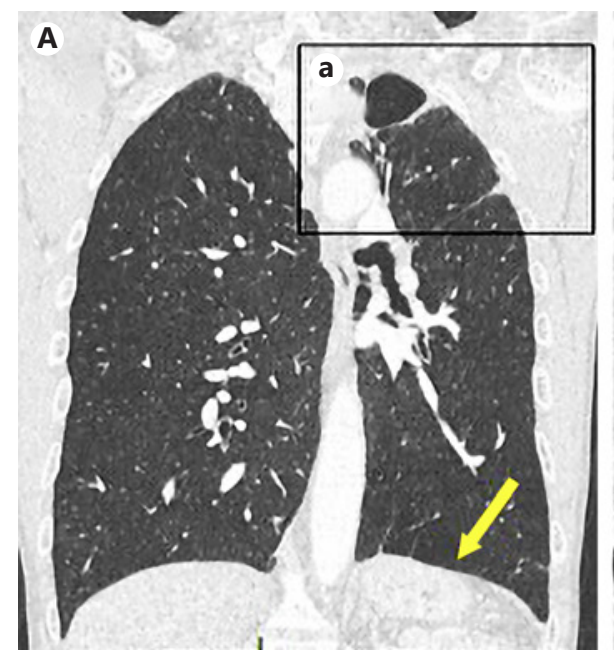

October 2007

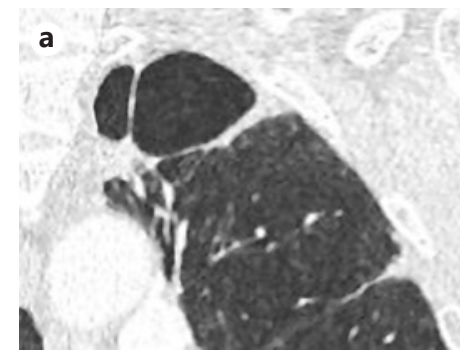

Fig. 4. A, a In October 2007, this 60-year-old nonsmoking patient was followed for TB sequelae. B, b In June 2010, he consulted for hemoptysis and the diagnosis of CPA was made; a few weeks later, treatment with voriconazole was started (200 mg twice daily). C, c In January 2011, hemoptysis was not renewed and a response assessment was performed. A, a On the CT scan of the chest of $10 / 2017$ (parenchymal window and frontal section), there is a thinwalled clarity of the right upper lobe. The yellow arrow indicates the position of the diaphragm, which is slightly elevated. $\mathbf{B}, \mathbf{b} \mathrm{On}$ the CT scan of the chest of $06 / 2010$, the cavity of the right upper

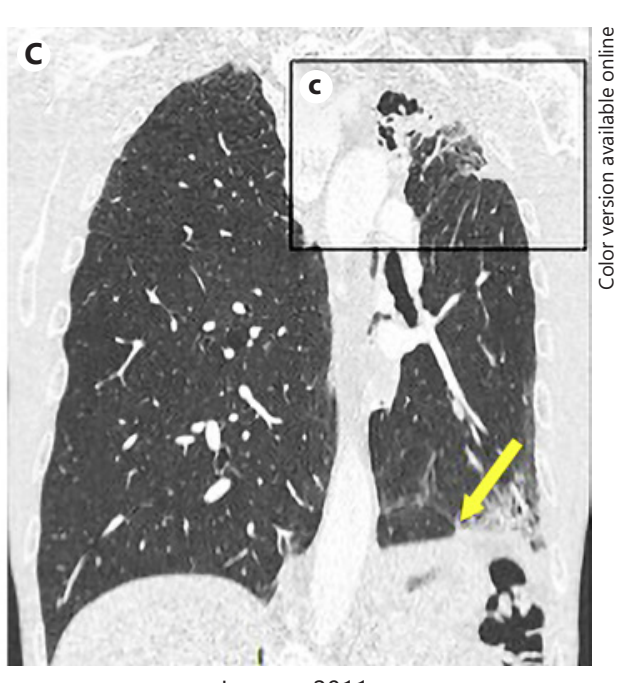

January 2011

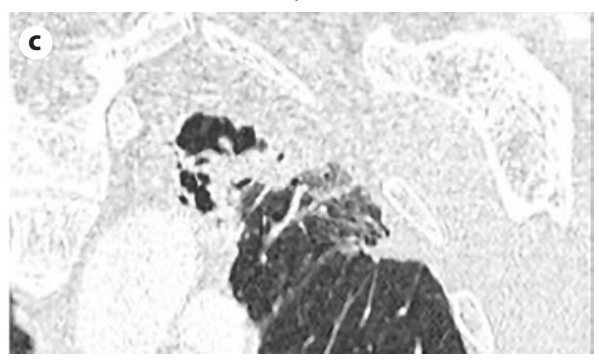

lobe is modified. It appears smaller (double white arrow), and there is a measurable thickening of the wall of the cavity (double black arrow). There is no intracavitary material. The lung parenchyma of the right upper lobe is partially condensed, explaining the rise of the diaphragmatic dome (yellow arrow). C, c After 6 months of treatment with voriconazole, the cavity remains with a thickened wall, but the volume of the cavity is reduced. The diaphragmatic dome is less elevated (yellow arrow) because the condensation of the parenchyma of the lungs has partially resolved.

ropean Respiratory Society (ERS) and the European Confederation of Medical Mycology (ECMM) and a strong recommendation in the guideline published by the Infectious Diseases Society of America (IDSA) for CPA treatment (Table 1) [2, 7]. In contrast, posaconazole has only a BII recommendation. Fluconazole has no anti-Aspergillus activity.

Itraconazole is the most commonly used Aspergillusactive azole. In the only randomized-controlled trial of azole therapy $(n=17)$ versus supportive care $(n=14)$, there was a significant benefit from a 6-month course of itraconazole $400 \mathrm{mg}$ daily [8]. Around a quarter of the treatment group improved and half remained stable. Unfortunately, around a quarter of patients deteriorated on stopping azole treatment, indicating that long-term therapy is needed for many patients. Three-quarters of patients in the control group had clinical or radiological deterioration. Voriconazole activity has been described in a single arm prospective study (22 patients with chronic cavitary pulmonary aspergillosis [CCPA] and 18 patients with chronic necrotizing pulmonary aspergillosis) [9]. The two studies used different outcomes and cannot be directly compared, but this study showed improvement in approximately a third of patients. In case of intolerance or toxicity, therapy may be changed from itraconazole to voriconazole or vice versa.

Antifungal treatment is recommended in symptomatic patients, although it is sometimes difficult to distin- 


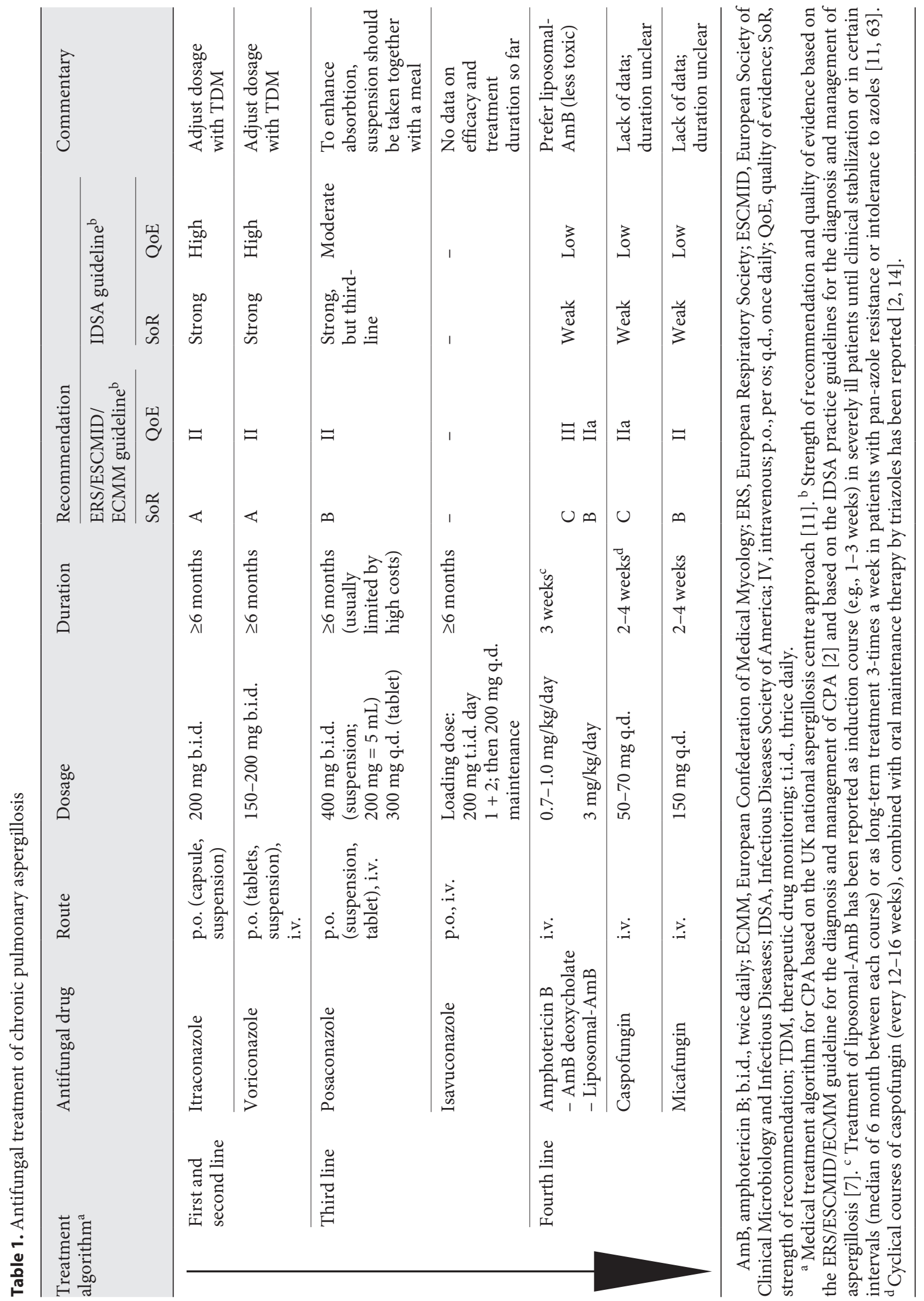


guish between CPA-associated symptoms and symptoms caused by the underlying respiratory disorder. Patients with CCPA and chronic fibrosing pulmonary aspergillosis usually benefit from antifungal treatment (Fig. 4), although treatment will not lead to relapse-free cure, but may improve symptoms and quality of life and slowdown or prevent disease progression [10]. The duration is generally recommended to be $\geq 6$ months, but should be based on an individual risk and benefit evaluation. Some patients, especially those with a mild immunosuppression, may need life-long antifungal treatment, if tolerated. Others, like patients with a simple/single aspergilloma or Aspergillus nodules, often do not respond to antifungal treatment. In these patients, a "wait and watch" strategy might be reasonable, but it should be considered that surgical resection could lead to relapsefree cure.

\section{Which Alternative Antifungal Treatment Can Be Used when First-Line Treatment with Itraconazole or Voriconazole Is Not Possible?}

In the event of toxicity and/or clinical or radiological ineffectiveness to a triazole, it is legitimate to propose the use of another triazole (itraconazole or voriconazole, then posaconazole and then isavuconazole) (Table 1) [11], after verifying that: (a) serum concentration of the antifungal drug was adequately monitored and (b) the Aspergillus strain was not resistant to the proposed triazole (in particular for itraconazole). In case of resistance or side effects to itraconazole or voriconazole, third-line treatment with posaconazole is possible, the activity of which has been described in a case series [12]. Posaconazole is generally better tolerated than either itraconazole or voriconazole [13]. The efficacy of posaconazole in CPA has been much less evaluated [12]; poor absorption of the posaconazole solution has been solved by the use of tablets. There are no data for isavuconazole. The UK National Aspergillosis Centre (www.nationalaspergillosiscentre.org.uk) approach considers (beside posaconazole) the novel triazole isavuconazole as third-line treatment, but comprehensive data about isavuconazole in CPA patients are lacking [11].

In the case of triazole toxicities, drug interaction problems, clinical failure or resistance of Aspergillus to triazoles, the therapeutic option is to use an i.v. antifungal (Table 1): (a) echinocandins or (b) amphotericin B, where liposomal is preferred to deoxycholate due to lower toxicity $[2,11]$. The use of a combination of tri- azole and caspofungin has been reported in a few cases of CPA complicating sarcoidosis [14]. Apart from intravenous micafungin, which was the subject of a phase III trial versus voriconazole [15], other treatments were only evaluated retrospectively $[2,3,11,16]$. The primary endpoint of the efficacy of these treatments varied widely from study to study, making interpretation of results difficult and unreliable [3]. The duration of treatment is limited by the need for i.v. treatment and the particularly renal toxicity of amphotericin B. An alternative to the i.v. administration of antifungals is the use of amphotericin B in local instillation by placing a catheter in the cavity rather than performing iterative bronchoscopies. More recently, it has also been proposed to administer liposomal amphotericin B by aerosol (see below) [17].

\section{Adverse Events, Drug-Drug Interaction and Therapeutic Drug Monitoring}

Adverse events occur frequently during itraconazole and voriconazole treatment. Hepatotoxicity is the most relevant adverse event during itraconazole treatment. Transient or permanent hearing loss has been described. Visual disturbances, hallucinations, cardiac complications, hepatobilliary disorders and skin and subcutaneous tissue disorders are the most relevant adverse events of voriconazole treatment [18]. Peripheral neuropathy has been reported with all azoles requiring cessation of treatment. A single retrospective study reported peripheral neuropathy in 17, 9 and $3 \%$ of patients taking itraconazole, voriconazole and posaconazole long-term, respectively [13]. In contrast, other treatment studies did not observe peripheral neuropathy as an adverse event [9]. QTc interval prolongation has been described for voriconazole, intraconazole and posaconazole, and may be potentiated if those azoles are administered together with quinolones [19-21]. In contrast, isavuconazole is associated with a shortened QTc interval with a mean decrease of $36.5 \pm 38.8 \mathrm{~ms}(7.4 \pm 5.8 \%)$ compared to the pre-isavuconazole electrocardiogram in a recent study [22]. Overall, isavuconazole has been shown in clinical trials and also a limited number of real life studies to be better tolerated than particularly voriconazole and itraconazole and may therefore be a viable oral alternative in patients experiencing adverse events [23].

Drug-drug interactions have to be taken into account before antifungal treatment with a broad-spectrum triazole can be initiated. Importantly, voriconazole is me- 
Table 2. ECIL-6 and ESCMID/ECMM/ERS 2017 recommendations for therapeutic drug monitoring for triazoles

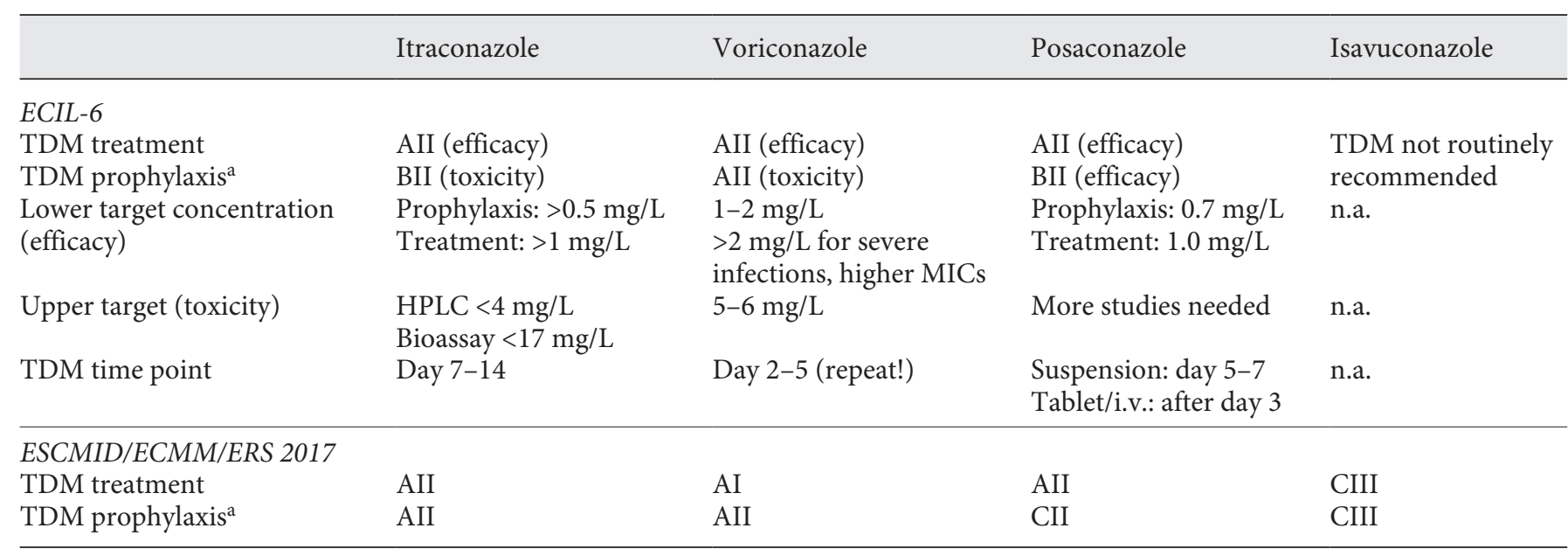

ECIL, European Conference on Infections in Leukemia; ECMM, the European Confederation of Medical Mycology; ESCMID, European Society of Clinical Microbiology and Infectious Diseases; ERS, the European Respiratory Society; HPLC, high performance liquid chromatography; i.v., intravenous; n.a., not applicable; MIC, minimal inhibitory concentration; TDM, therapeutic drug monitoring.

a Triazole prophylaxis refers to patients at risk for invasive aspergillosis.

tabolized over a variety of enzymes, mainly CYP2C19 and CYP2C9, but also CYP3A4/5, where voriconazole is a weak substrate. In contrast, itraconazole is strong substrate of only CYP3A4/5. As a result, there are a number of relevant drug-drug interactions over CYP3A4/5 for both itraconazole and voriconazole including rifamycins, warfarin, clopidogrel, immunosuppressive drugs, highdose ritonavir, etravirine and efavirenz, simvastatin, where coadministration is contraindicated or close monitoring is required for both itraconazole and voriconazole [24]. Also macrolides have been shown to increase plasma levels of itraconazole and voriconazole, and close therapeutic drug monitoring (TDM) may be required to avoid toxicity in patients receiving both. Especially the drug-drug interaction between rifamycins and triazoles has to be considered since patients with pulmonary tuberculosis have an increased risk to develop CPA coinfection. Concomitant use of rifamycins and triazoles should be avoided in patients with drug-susceptible pulmonary tuberculosis with CPA coinfection or in patients with nontuberculous mycobacterial pulmonary diseases [24, 25]. Echinocandins may be considered as alternative antifungal drugs, but evidence is limited [26]. When voriconazole or itraconazole are administered with some immunosuppressant drugs, extreme caution is required, with dosages of cyclosporine and tacrolimus requiring significant reduction. Importantly, sirolimus is contraindicated in those receiving voriconazole, and preemptive sirolimus dose reduction is required in those receiving itraconazole [27]. In those patients, isavuconazole, which has fewer drug-drug interactions with immunosuppressive drugs, may be a better alternative for treatment of CPA [23].

Given the high intra- and interpatient variability of voriconazole and itraconazole plasma concentrations, TDM has been strongly recommended by the European Conference on Infections in Leukemia-6 (ECIL-6) and the ESCMID/ECMM/ERS 2017 guidelines for both triazoles, to ensure efficacy and avoid toxicity (Table 2) [28, 29]. For voriconazole, the first trough level should be obtained between days 2 and 5 and, given the high intrapatient variability, optimally repeated once per week, to ensure plasma concentrations remain within the small therapeutic window of $>1 \mathrm{mg} / \mathrm{L}$ and $<6 \mathrm{mg} / \mathrm{L}[30,31]$. For itraconazole, the first trough level is recommended between days 7 and 14 , and the target is $>1 \mathrm{mg} / \mathrm{L}$ and $<4$ $\mathrm{mg} / \mathrm{L}$ [28]. TDM is also recommended for posaconazole, in particular the posaconazole oral suspension, with the first trough level between days 5 and 7; however, intrapatient variability is significantly lower compared to voriconazole, and therefore levels do not have to be repeated as frequently. In contrast, TDM is not routinely recommended for isavuconazole, which has a much lower intraand interpatient variability and no clear exposure-response relationship. 
Table 3. Clinical breakpoints of amphotericin B and azoles drugs according the EUCAST

\begin{tabular}{|c|c|c|c|c|c|c|c|c|c|c|}
\hline Antifungal agent & \multicolumn{10}{|c|}{ MIC breakpoint, mg/L } \\
\hline Itraconazole & 1 & 2 & 1 & 2 & 1 & 2 & - & - & 1 & 2 \\
\hline Voriconazole & 1 & 2 & - & - & - & - & - & - & - & - \\
\hline Amphotericin B & 1 & 2 & - & - & - & - & 1 & 2 & - & - \\
\hline
\end{tabular}

EUCAST, European Committee on Antimicrobial Susceptibility Testing; MIC, minimal inhibitory concentration; R, resistant; S, susceptible.

\section{What Is the Role of Drug Resistance Testing in the Management of CPA Patients?}

The emergence of antifungal resistance is becoming a problem for the treatment of CPA [32]. Azole resistance in A. fumigatus has been increasingly described in the last decade, being associated with poorer outcomes [33]. Prolonged treatment in patients with chronic disease seems to play a major role in the emergence of antifungal resistance $[34,35]$. CPA belongs to these clinical situations with a persistence of the fungal pathogen for weeks to years before the diagnosis could be made followed by long-term antifungal treatment that may poorly penetrate into pulmonary cavities or fibrosis. Antifungal resistance in the context of CPA is mainly a concern in azole derivatives, because they exercise the most common selective pressure as first-line therapy. Acquired resistance to amphotericin B or echinocandins is very scarce with Aspergillus.

Despite the increasing interest in CPA, the true prevalence of azole resistance in A. fumigatus, the main causative species of CPA, and the risk to see the emergence of such resistance are still unknown. A prevalence of azole resistance of A. fumigatus between 2 and $54 \%$ has been reported, but prospective studies are required for a better estimation [36]. While not clearly demonstrated for CPA, there is probably a link between in vitro resistance and clinical failure [33]. Thus, a mycological examination of respiratory specimens at every outpatient visit is advisable both for analyzing the mycological efficacy of the antifungal regimen, and, in the case of positive culture, to examine the antifungal in vitro susceptibility (AFST). Although respiratory specimens from the lower respiratory tract are preferred for the diagno- sis of infection, it has been demonstrated that sputum samples could also be used [37]. Due to the low sensitivity of culture, several efforts have been directed to obtain better results when culturing respiratory samples. Culture of multiple samples, use of higher volumes and undiluted samples have been associated with a higher yield [38-40].

Standardized reference methods for AFST of Aspergillus clinical isolates and clinical breakpoints are available by the European Committee on Antimicrobial Susceptibility Testing (EUCAST) and the Clinical Laboratory Standards Institute (CLSI) organizations [41]. Both yield similar results and could be used to detect resistance in Aspergillus. Species-specific breakpoints by the EUCAST method can be found in Table 3 and should be used to define resistance in Aspergillus. Commercially available agar-based methods have been proven to be useful to detect azole resistance in Aspergillus and could be an alternative for clinical laboratories [42, 43].

Although other mechanisms of resistance have been found, azole resistance in Aspergillus is mainly linked with mutations in the cyp51A gene coding for the lanosterol 14a-demethylase, an enzyme involved in the synthesis of ergosterol, the main sterol of the fungal membrane (Fig. 5) [44]. Different molecular mechanisms have been described associated with various patterns of azole resistance. Molecular methods to detect $c y p 51 \mathrm{~A}$-mediated resistance in A. fumigatus have been developed [45-49]. Some of these methods have been directly applied in clinical specimens and could therefore be good alternatives in infections where a culture is not available. In addition, positive cultures during antifungal therapy could indicate azole resistance (Fig. 6) [34]. 


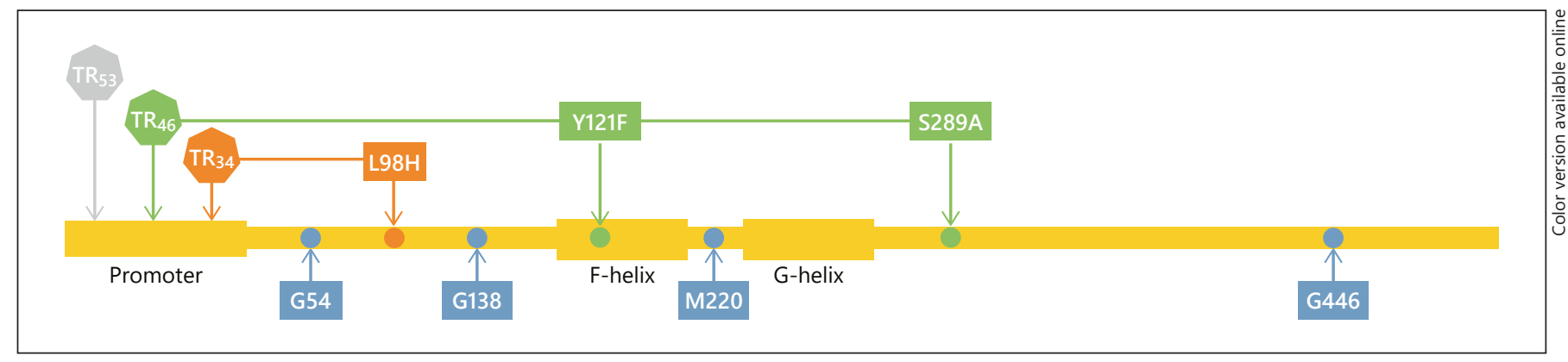

Fig. 5. Cyp51A modifications leading to azole resistance in A. fumigatus. Upper part represents mutations associated with TR (tandem repeats) causing overexpression of Cyp51A. Lower part represents positions at which main point mutations leading to azole resistance occur.

Fig. 6. E-test plate of an A. fumigatus susceptible wild type strain $(\mathbf{A})$ and a voriconazole-resistant $\mathrm{TR}_{34} / \mathrm{L} 98 \mathrm{H}$ mutant strain (B).
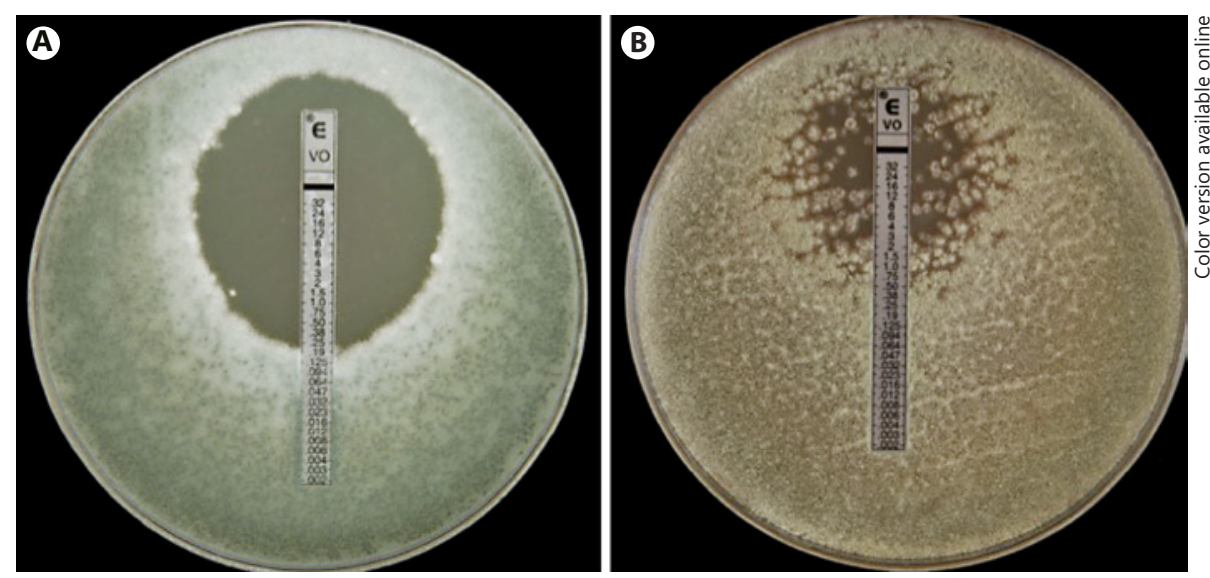

\section{Are There New Drugs or Drug Applications in the Pipeline?}

Isavuconazole is a novel triazole agent with a wide spectrum of antifungal activity. The ECIL- 6 guideline recommends it as first-line therapy for invasive aspergillosis in patients with hematological malignancies (AI AII recommendation), and the IDSA recommends it as an alternative treatment $[7,28]$. The main advantages of isavuconazole compared to voriconazole are: (a) reduced nephrotoxicity in patients given intravenous therapy; (b) no required dose adjustments in patients with renal and hepatic dysfunction; (c) fewer drug-drug interactions, only interactions with CYP3A4 inducers or inhibitors; and (d) no contribution to a prolonged QTc interval (isavuconazole shortens the QTc interval) $[18,22,50]$.

For pan-azole resistance, short courses of intravenous liposomal amphotericin B (LAmB) or micafungin are fourth-line therapy, keeping in mind amphotericin $\mathrm{B}$ nephrotoxicity [11]. Authors have reported cases of bronchopulmonary aspergillosis in which LAmB inhalation served as an alternative treatment for aspergillosis pulmonary diseases $[16,51]$. Inhaled antifungal treatment using LAmB could present several advantages: (a) heightening antifungal lung tissue concentration in devascularized sites; (b) limiting drug interactions and side effects due to lack of plasmatic efflux; and (c) potentially leading to improved eradication of Aspergillus and to optimized treatment duration, thereby limiting the risk of emergence of treatment-resistant Aspergillus strains [16]. The major medico-economic impact of therapeutic failures should motivate efforts towards implementation of new therapeutic strategies aimed at drug formulations and/or administration methods (such as nebulized route) to heighten lung tissue concentration, limit drug interactions, reduce side effects, and optimize treatment duration, which represent the main predictive factors for development of resistance and therapeutic failure $[2,52]$. 


\section{In Which Patients Should Surgery Be Considered?}

The decision to proceed to surgery is usually individualized. As CPA patients have frequently relevant comorbidities, a thoroughly preoperative evaluation (functional status, nutritional assessment, cardiopulmonary function) is obligatory in every case to reduce postoperative complications. Surgery is considered as treatment option in the following specific situations [2]:

1. Symptomatic patients with simple aspergillomas can be managed surgically (Fig. 2). The postoperative prognosis is good. Furthermore, minimal-invasive approaches may be appropriate in peripheral aspergilloma without infiltration of the hilum [53-55]. In immunocompetent patients with simple aspergilloma, adjuvant antifungal therapy is not routinely needed $[56,57]$.

2. In patients with CCPA, surgery should be considered if long-term antifungal treatment is ineffective, not tolerable or unfeasible (multi-azole resistance). However, postoperative complications are more frequent than in simple aspergillomas (e.g., persistent air-leak, wound infection, bronchopleural fistula (Fig. 3B), respiratory failure) $[54,55,58-60]$. A favorable outcome and a low recurrence rate depend on a careful patient selection and the ability to fully resect the affected lung parenchyma without fungal spillage into the pleural space. An experienced surgical team in CCPA surgery should perform the resection. Preoperative antifungal therapy should be considered in any complex situation (starting 2 weeks before resection). The duration of postoperative antifungal therapy has to be individualized. In case of intraoperative spillage, antifungal therapy should be given for at least 2 months. Long-term therapy may be required after incomplete resection $[55,61]$.

3. In CPA-associated hemoptysis, surgical interventions is a last-line therapy (Fig. 3B). Patients with mild hemoptysis are primarily treated with tranexamic acid and antifungal therapy. Moderate or severe cases should additionally be managed with bronchial artery catheter embolization. However, if these interventions are not effective, elective definite surgery should be considered [62].

\section{Conclusion}

$\mathrm{CPA}$ is a challenging fungal infection associated with high morbidity and mortality. Clinical presentation is nonspecific, and diagnosis if often delayed or missed. Clinical studies are scarce, and efficient treatment and management strategies need to be optimized. Surgery should be considered when possible, especially in cases of localized disease, but is not available for many patients with progressive disease or complicated comorbidities. Antifungal therapy leads to clinical stability or improvement, but the response rate is still moderate (Fig. 1). Oral therapy with triazoles (itraconazole or voriconazole) is the first-line treatment, but can be complicated by development of toxicity and/or antifungal resistance. Other therapeutic strategies like intravenous amphotericin B or micafungin can be used in short regimens, but are suboptimal for long-term treatment. Isavuconazole and nebulized amphotericin B are currently interesting alternatives that have to be evaluated in this population. Current CPA treatments can benefit patients, but have many drawbacks. Optimal treatment is yet to be established in $\mathrm{CPA}$ patients and warrants further investigation. New antifungal drugs, therapeutic strategies and a better optimization of the currently available ones need to be further evaluated through clinically oriented research in this neglected fungal disease.

\section{Disclosure Statement}

The authors have no conflicts of interest to declare.

\section{References}

1 Salzer HJ, Heyckendorf J, Kalsdorf B, Rolling $\mathrm{T}$, Lange C: Characterization of patients with chronic pulmonary aspergillosis according to the new ESCMID/ERS/ECMM and IDSA guidelines. Mycoses 2017;60:136-142.

2 Denning DW, Cadranel J, Beigelman-Aubry C, Ader F, Chakrabarti A, et al: Chronic pulmonary aspergillosis: rationale and clinical guidelines for diagnosis and management. Eur Respir J 2016;47:45-68.
3 Godet C, Alastruey-Izquierdo A, Flick H, Hennequin C, Mikilps-Mikgelbs R, Munteanu O, Page I, Seidel D, Salzer HJF; CPAnet: A CPAnet consensus statement on research priorities for chronic pulmonary aspergillosis: a neglected fungal infection that requires attention. J Antimicrob Chemother 2018;73:280286.
4 Koyama K, Ohshima N, Suzuki J, Kawashima M, Takeda K, Ando T, Sato R, Nagai H, Matsui H, Ohta K: Recurrence of chronic pulmonary aspergillosis after discontinuation of maintenance treatment by antifungal triazoles. J Infect Chemother 2014;20:375-379.

5 Godet C, Laurent F, Bergeron A, Ingrand P, et al: CT imaging assessment of response to treatment in chronic pulmonary aspergillosis. Chest 2016;150:139-147. 
6 Howard SJ, Pasqualotto AC, Anderson MJ, Leatherbarrow H, Albarrag AM, Harrison E, Gregson L, Bowyer P, Denning DW: Major variations in Aspergillus fumigatus arising within aspergillomas in chronic pulmonary aspergillosis. Mycoses 2013;56:434-441.

7 Patterson TF, Thompson GR 3rd, Denning DW, Fishman JA, Hadley S, Herbrecht R, Kontoyiannis DP, Marr KA, Morrison VA, Nguyen MH, Segal BH, Steinbach WJ, Stevens DA, Walsh TJ, Wingard JR, Young JA, Bennett JE: Practice guidelines for the diagnosis and management of aspergillosis: 2016 update by the Infectious Diseases Society of America. Clin Infect Dis 2016;63:e1-e60.

8 Agarwal R, Vishwanath G, Aggarwal AN, Garg M, Gupta D, Chakrabarti A: Itraconazole in chronic cavitary pulmonary aspergillosis: a randomised controlled trial and systematic review of literature. Mycoses 2013;56: 559-570.

9 Cadranel J, Philippe B, Hennequin C, Bergeron A, Bergot E, Bourdin A, Cottin V, Jeanfaivre T, Godet C, Pineau M, Germaud P: Voriconazole for chronic pulmonary aspergillosis: a prospective multicenter trial. Eur J Clin Microbiol Infect Dis 2012;31:3231-3239.

10 Al-Shair K, Atherton GT, Harris C, Ratcliffe L, Newton PJ, Denning DW: Long-term antifungal treatment improves health status in patients with chronic pulmonary aspergillosis: a longitudinal analysis. Clin Infect Dis 2013;57:828-835.

11 Maghrabi F, Denning DW: The management of chronic pulmonary aspergillosis: the UK National Aspergillosis Centre approach. Curr Fungal Infect Rep 2017;11:242-251.

12 Felton TW, Baxter C, Moore CB, Roberts SA, Hope WW, Denning DW: Efficacy and safety of posaconazole for chronic pulmonary aspergillosis. Clin Infect Dis 2010;51:13831391.

13 Baxter CG, Marshall A, Roberts M, Felton TW, Denning DW: Peripheral neuropathy in patients on long-term triazole antifungal therapy. J Antimicrob Chemother 2011;66: 2136-2139.

14 Keir GJ, Garfield B, Hansell DM, Loebinger MR, Wilson R, Renzoni EA, Wells AU, Maher TM: Cyclical caspofungin for chronic pulmonary aspergillosis in sarcoidosis. Thorax 2014; 69:287-288

15 Kohno S, Izumikawa K, Ogawa K, Kurashima A, Okimoto N, Amitani R, Kakeya H, Niki Y, Miyazaki Y; Japan Chronic Pulmonary Aspergillosis Study Group: Intravenous micafungin versus voriconazole for chronic pulmonary aspergillosis: a multicenter trial in Japan. J Infect 2010;61:410-418.

16 Godet C, Philippe B, Laurent F, Cadranel J: Chronic pulmonary aspergillosis: an update on diagnosis and treatment. Respiration 2014; $88: 162-174$.
17 Godet C, Goudet V, Laurent F, Le Moal G, Gounant V, Frat JP, Cateau E, Roblot F, Cadranel J: Nebulised liposomal amphotericin B for Aspergillus lung diseases: case series and literature review. Mycoses 2015;58:173180.

18 Maertens JA, Raad II, Marr KA, Patterson TF, et al: Isavuconazole versus voriconazole for primary treatment of invasive mould disease caused by Aspergillus and other filamentous fungi (SECURE): a phase 3, randomised-controlled, non-inferiority trial. Lancet 2016;387: 760-769.

19 Cornely OA, Maertens J, Winston DJ, Perfect J, Ullmann AJ, Walsh TJ, Helfgott D, Holowiecki J, Stockelberg D, Goh YT, Petrini M, Hardalo C, Suresh R, Angulo-Gonzalez D: Posaconazole versus fluconazole or itraconazole prophylaxis in patients with neutropenia. N Engl J Med 2007;356:348-359.

20 Alkan Y, Haefeli WE, Burhenne J, Stein J, Yaniv I, Shalit I: Voriconazole-induced QT interval prolongation and ventricular tachycardia: a non-concentration-dependent adverse effect. Clin Infect Dis 2004;39:e49-e52.

21 Zeuli JD, Wilson JW, Estes LL: Effect of combined fluoroquinolone and azole use on QT prolongation in hematology patients. Antimicrob Agents Chemother 2013;57:11211127.

22 Mellinghoff SC, Bassetti M, Dorfel D, Hagel S, Lehners N, Plis A, Schalk E, Vena A, Cornely OA: Isavuconazole shortens the QTc interval. Mycoses 2018;61:256-260.

23 Hoenigl M, Prattes J, Neumeister P, Wolfler A, Krause R: Real-world challenges and unmet needs in the diagnosis and treatment of suspected invasive pulmonary aspergillosis in patients with haematological diseases: an illustrative case study. Mycoses 2018;61:201205.

24 Moon SM, Park HY, Jeong BH, Jeon K, Lee SY, Koh WJ: Effect of rifampin and rifabutin on serum itraconazole levels in patients with chronic pulmonary aspergillosis and coexisting nontuberculous mycobacterial infection. Antimicrob Agents Chemother 2015;59:663665.

25 Gubbins PO, Heldenbrand S: Clinically relevant drug interactions of current antifungal agents. Mycoses 2010;53:95-113.

26 Kohno S, Izumikawa K, Kakeya H, Miyazaki Y, Ogawa K, Amitani R, Niki Y, Kurashima A: Clinical efficacy and safety of micafungin in Japanese patients with chronic pulmonary aspergillosis: a prospective observational study. Med Mycol 2011;49:688-693.

27 Said A, Garnick JJ, Dieterle N, Peres E, Abidi MH, Ibrahim RB: Sirolimus-itraconazole interaction in a hematopoietic stem cell transplant recipient. Pharmacotherapy 2006;26: 289-295.
28 Tissot F, Agrawal S, Pagano L, Petrikkos G, Groll AH, Skiada A, Lass-Florl C, Calandra T, Viscoli C, Herbrecht R: ECIL-6 guidelines for the treatment of invasive candidiasis, aspergillosis and mucormycosis in leukemia and hematopoietic stem cell transplant patients. Haematologica 2017;102:433-444.

29 Ullmann AJ, Aguado JM, Arikan-Akdagli S, Denning DW, et al: Diagnosis and management of Aspergillus diseases: executive summary of the 2017 ESCMID-ECMM-ERS guideline. Clin Microbiol Infect 2018;24(suppl 1):e1-e38.

30 Hoenigl M, Duettmann W, Raggam RB, Seeber K, Troppan K, Fruhwald S, Prueller F, Wagner J, Valentin T, Zollner-Schwetz I, Wolfler A, Krause R: Potential factors for inadequate voriconazole plasma concentrations in intensive care unit patients and patients with hematological malignancies. Antimicrob Agents Chemother 2013;57:3262-3267.

31 Pascual A, Calandra T, Bolay S, Buclin T, Bille J, Marchetti O: Voriconazole therapeutic drug monitoring in patients with invasive mycoses improves efficacy and safety outcomes. Clin Infect Dis 2008;46:201-211.

32 Perlin DS, Rautemaa-Richardson R, Alastruey-Izquierdo A: The global problem of antifungal resistance: prevalence, mechanisms, and management. Lancet Infect Dis 2017; 17:e383-e392.

33 van der Linden JW, Snelders E, Kampinga GA, Rijnders BJ, Mattsson E, Debets-Ossenkopp YJ, Kuijper EJ, Van Tiel FH, Melchers WJ, Verweij PE: Clinical implications of azole resistance in Aspergillus fumigatus, the Netherlands, 2007-2009. Emerg Infect Dis 2011; 17:1846-1854.

34 Howard SJ, Cerar D, Anderson MJ, Albarrag A, Fisher MC, Pasqualotto AC, Laverdiere M, Arendrup MC, Perlin DS, Denning DW: Frequency and evolution of azole resistance in Aspergillus fumigatus associated with treatment failure. Emerg Infect Dis 2009;15:10681076.

35 Rivero-Menendez O, Alastruey-Izquierdo A, Mellado E, Cuenca-Estrella M: Triazole resistance in Aspergillus spp: a worldwide problem? J Fungi (Basel) 2016;2.

36 Godet C, Alastruey-Izquierdo A, Flick H, Hennequin C, Mikilps-Mikgelbs R, Munteanu O, Page I, Seidel D, Salzer HJF: A CPAnet consensus statement on research priorities for chronic pulmonary aspergillosis: a neglected fungal infection that requires attention. J Antimicrob Chemother 2018;73:280-286.

37 Escribano P, Marcos-Zambrano LJ, Pelaez T, Munoz P, Padilla B, Bouza E, Guinea J: Sputum and bronchial secretion samples are equally useful as bronchoalveolar lavage samples for the diagnosis of invasive pulmonary aspergillosis in selected patients. Med Mycol 2015;53:235-240.

38 Chakrabarti A, Sethi S, Raman DS, Behera D: Eight-year study of allergic bronchopulmonary aspergillosis in an Indian teaching hospital. Mycoses 2002;45:295-299.
Treatment of Chronic Pulmonary Aspergillosis
Respiration 2018;96:159-170 DOI: $10.1159 / 000489474$ 
39 Fraczek MG, Kirwan MB, Moore CB, Morris J, Denning DW, Richardson MD: Volume dependency for culture of fungi from respiratory secretions and increased sensitivity of Aspergillus quantitative PCR. Mycoses 2014;57:69-78.

40 Pashley CH, Fairs A, Morley JP, Tailor S, Agbetile J, Bafadhel M, Brightling CE, Wardlaw AJ: Routine processing procedures for isolating filamentous fungi from respiratory sputum samples may underestimate fungal prevalence. Med Mycol 2012;50:433-438.

41 Bernal-Martinez L, Alastruey-Izquierdo A, Cuenca-Estrella M: Diagnostics and susceptibility testing in Aspergillus. Future Microbiol 2016;11:315-328.

42 Buil JB, van der Lee HAL, Rijs A, Zoll J, Hovestadt J, Melchers WJG, Verweij PE: Singlecenter evaluation of an agar-based screening for azole resistance in Aspergillus fumigatus by using VIPcheck. Antimicrob Agents Chemother 2017;61:e01250-17.

43 Idelevich EA, Gross U, Becker K, Bader O: Comparative evaluation of different gradient diffusion tests for detection of azole resistance in Aspergillus fumigatus. Diagn Microbiol Infect Dis 2018;91:52-54.

44 Chowdhary A, Sharma C, Hagen F, Meis JF: Exploring azole antifungal drug resistance in Aspergillus fumigatus with special reference to resistance mechanisms. Future Microbiol 2014;9:697-711.

45 Bernal-Martinez L, Gil H, Rivero-Menendez O, Gago S, Cuenca-Estrella M, Mellado E, Alastruey-Izquierdo A: Development and validation of a high-resolution melting assay to detect azole resistance in Aspergillus fumigatus. Antimicrob Agents Chemother 2017; 61:e01083-17.

46 Chong GM, van der Beek MT, von dem Borne PA, Boelens J, Steel E, Kampinga GA, Span LF, Lagrou K, Maertens JA, Dingemans GJ, Gaajetaan GR, van Tegelen DW, Cornelissen JJ, Vonk AG, Rijnders BJ: PCR-based detection of Aspergillus fumigatus cyp 51a muta- tions on bronchoalveolar lavage: a multicentre validation of the AsperGenius assay ${ }^{\circledR}$ in 201 patients with haematological disease suspected for invasive aspergillosis. J Antimicrob Chemother 2016;71:3528-3535.

47 Garcia-Effron G, Dilger A, Alcazar-Fuoli L, Park S, Mellado E, Perlin DS: Rapid detection of triazole antifungal resistance in Aspergillus fumigatus. J Clin Microbiol 2008;46:1200-1206.

48 Klaassen CH, de Valk HA, Curfs-Breuker IM, Meis JF: Novel mixed-format real-time PCR assay to detect mutations conferring resistance to triazoles in Aspergillus fumigatus and prevalence of multi-triazole resistance among clinical isolates in the Netherlands. J Antimicrob Chemother 2010;65:901-905.

49 Zhao Y, Garnaud C, Brenier-Pinchart MP, Thiebaut-Bertrand A, Saint-Raymond C, Camara B, Hamidfar R, Cognet O, Maubon D, Cornet M, Perlin DS: Direct molecular diagnosis of aspergillosis and cyp 51a profiling from respiratory samples of French patients. Front Microbiol 2016;7:1164.

50 Keirns J, Desai A, Kowalski D, Lademacher C, Mujais S, Parker B, Schneidkraut MJ, Townsend R, Wojtkowski T, Yamazaki T, Yen M, Kowey PR: QT interval shortening with isavuconazole: in vitro and in vivo effects on cardiac repolarization. Clin Pharmacol Ther 2017;101:782-790

51 Birsan T, Taghavi S, Klepetko W: Treatment of Aspergillus-related ulcerative tracheobronchitis in lung transplant recipients. J Heart Lung Transplant 1998;17:437-438.

52 Verweij PE, Zhang J, Debets AJM, Meis JF, van de Veerdonk FL, Schoustra SE, Zwaan BJ, Melchers WJG: In-host adaptation and acquired triazole resistance in Aspergillus fumigatus: a dilemma for clinical management. Lancet Infect Dis 2016;16:e251-e260.

53 Chen QK, Chen C, Chen XF, Jiang GN: Video-assisted thoracic surgery for pulmonary aspergilloma: a safe and effective procedure. Ann Thorac Surg 2014;97:218-223.
54 El Hammoumi MM, Slaoui O, El Oueriachi F, Kabiri EH: Lung resection in pulmonary aspergilloma: experience of a Moroccan center. BMC Surg 2015;15:114.

55 Farid S, Mohamed S, Devbhandari M, Kneale M, Richardson M, Soon SY, Jones MT, Krysiak P, Shah R, Denning DW, Rammohan $\mathrm{K}$ : Results of surgery for chronic pulmonary aspergillosis, optimal antifungal therapy and proposed high risk factors for recurrence - a national centre's experience. J Cardiothorac Surg 2013;8:180.

56 Benhamed L, Woelffle D: Adjuvant antifungal therapy after pulmonary surgery for aspergilloma: is it useful? Interact Cardiovasc Thorac Surg 2014;18:835-837.

57 Sagan D, Gozdziuk K: Surgery for pulmonary aspergilloma in immunocompetent patients: no benefit from adjuvant antifungal pharmacotherapy. Ann Thorac Surg 2010;89:16031610.

58 Chen QK, Jiang GN, Ding JA: Surgical treatment for pulmonary aspergilloma: a 35-year experience in the Chinese population. Interact Cardiovasc Thorac Surg 2012;15:77-80.

59 Kim YT, Kang MC, Sung SW, Kim JH: Good long-term outcomes after surgical treatment of simple and complex pulmonary aspergilloma. Ann Thorac Surg 2005;79:294-298.

60 Regnard JF, Icard P, Nicolosi M, Spagiarri L, Magdeleinat P, Jauffret B, Levasseur P: Aspergilloma: a series of 89 surgical cases. Ann Thorac Surg 2000;69:898-903.

61 Kosmidis C, Denning DW: The clinical spectrum of pulmonary aspergillosis. Thorax 2015;70:270-277.

62 Corr P: Management of severe hemoptysis from pulmonary aspergilloma using endovascular embolization. Cardiovasc Intervent Radiol 2006;29:807-810.

63 Newton PJ, Harris C, Morris J, Denning DW: Impact of liposomal amphotericin B therapy on chronic pulmonary aspergillosis. J Infect 2016;73:485-495. 